\title{
Características clínicas de los pacientes con Fibrilación Auricular
}

Clinical characteristics of patients with Atrial Fibrillation

Balarezo Arias Fernando Xavier ${ }^{1}$, Anilema Cóndor Raúl Daniel ${ }^{1}$

VOLUMEN 39 | No 1 I SEPTIEMBRE 2021

FECHA DE RECEPCIÓN: 10/11/2020 FECHA DE APROBACIÓN: 21/07/2021 FECHA PUBLICACIÓN: 16/09/2021

1. Médico en libre ejercicio. Cuenca - Ecuador

\section{Artículo Original original Article}

DOI: https://doi.org/10.18537/RFCM.39.01.02

Correspondencia:

balarezo.fernandolahotmail.com

Dirección:

Miguel Narea y Antonio de Ricaurte

Código Postal:

010108

Teléfono/Celular:

$072890424-0986493274$

Cuenca - Ecuador

\section{RESUMEN}

Objetivo: caracterizar la Fibrilación Auricular (FA), frecuencia de sus comorbilidades, riesgo tromboembólico y las estrategias terapéuticas en pacientes del Hospital José Carrasco Arteaga de la ciudad de Cuenca.

Metodología: estudio descriptivo con un universo de 297 pacientes diagnosticados de FA del Hospital José Carrasco Arteaga, durante el año 2017. La información se recolectó en un formulario elaborado por los autores y se analizó con los programas PSPP Free Software Foundation, y Epidat 4.1 mediante distribuciones de frecuencia, porcentajes y media.

Resultados: el $52.5 \%$ de la población estudiada fueron hombres; la comorbilidad más frecuente fue la hipertensión arterial en el $50.5 \%$ de los casos; la FA no valvular representó al $82.5 \%$ de los casos, el riesgo tromboembólico fue alto en el $77.1 \%$ de los pacientes con FA no valvular; la anticoagulación fue la estrategia terapéutica en el $67 \%$ de pacientes.

Conclusión: las características clínicas de los pacientes con FA de la localidad resultan congruentes con las de poblaciones similares. La mayoría son hombres, adultos mayores e hipertensos. El riesgo embólico alto fue predominante, siendo la warfarina el fármaco más usado.

Palabras clave: fibrilación auricular, tromboembolia, anticoagulante. 


\section{ABSTRACT}

Objective: to characterize Atrial Fibrillation (AF) to determine the frequency of the main comorbidities of atrial fibrillation, the thromboembolic risk and the main therapeutic strategy in patients of the Hospital "José Carrasco Arteaga" in Cuenca city.

Methodology: it is a descriptive study with a universe of 297 patients with a diagnosis of Atrial Fibrillation at the José Carrasco Arteaga Hospital, during 2017. The information was collected in a form prepared by the authors and analyzed with the PSPP Free Software Foundation, and Epidat programs 4.1 using frequency, percentage and mean distributions.

Results: a total of $52.5 \%$ of the studied population were men; the most frequent comorbidity was arterial hypertension in $50.5 \%$ of the cases; nonvalvular Atrial Fibrillation represented $82.5 \%$ of the cases, the thromboembolic risk was high in $77.1 \%$ of the patients with non-valvular atrial fibrillation; anticoagulation was the therapeutic strategy in $67 \%$ of patients.

Conclusion: the clinical characteristics of patients with $\mathrm{AF}$ in our city are consistent with those of similar populations. Most are men, older adults, and hypertensive. High embolic risk was predominant, consequently warfarin was the most used drug.

Key words: atrial fibrillation, thromboembolism, anticoagulants.

\section{INTRODUCCIÓN}

De acuerdoa la Organización Mundial de la Salud (OMS) las enfermedades cardiovasculares son la principal causa de muerte en el mundo, es así que en el 2015 causaron 17.7 millones de muertes que representó el $31 \%$ de todas las registradas en el planeta y más del $80 \%$ se produjeron en países de bajos y medianos ingresos como el Ecuador ${ }^{1}$.

Este estudio está enfocado en la FA, una taquiarritmia supraventricular caracterizada por la activación auricular desorganizada que ocasiona contracciones auriculares inadecuadas ${ }^{2}$. Es la arritmia más frecuente a nivel mundial pues de acuerdo a los resultados el estudio Framingham para el año 2013 afectó a 33 millones de personas cifra que representa al $0.5 \%$ de la población ${ }^{3}$.

Se estima que en Europa la prevalencia en la población general es entre el $0.2-2 \%$ y en mayores de 50 años de hasta el $5.5 \%$ en tanto, Estados Unidos ha estimado una prevalencia del $1 \%$ de la población general y en adultos mayores de 85 años entre el $11 \%$ y $12 \%$.

En poblaciones semejantes a la local, como Colombia, Castro et $\mathrm{al}^{6}$ estimaron una prevalencia global de FA de $4.8 \%$ en pacientes hospitalizados, en Paraguay se ha evidenciado una prevalencia de $11.8 \%$ con un mayor predominio del sexo masculino ${ }^{7}$.

La FA puede aparecer en corazones estructuralmente normales (fibrilación aislada) o puede deberse a cardiopatías coexistentes (insuficiencia cardiaca, cardiopatía isquémica, hipertensiva, reumática, entre otras) que pudieran ser su única manifestación clínica; de igual manera, el riesgo embólico no es homogéneo en los pacientes y depende de factores individuales propios del individuo ${ }^{8}$.

El conocimiento sobre FA se encuentra en fase de estudio y desarrollo, sin embargo, pese al incremento en su prevalencia e incidencia, en Ecuador al igual que el resto de Latinoamérica, por falta de investigación no se dispone de datos adecuados sobre esta patología, por ello, este estudio tiene como objetivo determinar las principales características clínicas y comorbilidades que presentan los pacientes con FA de la institución.

\section{METODOLOGÍA}

Estudio descriptivo que se desarrolló desde enero hasta diciembre del año 2017; se incluyó las historias clínicas de pacientes mayores de 18 años con diagnóstico confirmado de FA y se excluyó aquellas historias clínicas incompletas o incongruentes. La recolección de los datos se llevó a cabo por los autores para evitar sesgos y errores, el instrumento fue el formulario que constó con las variables del estudio. Para determinar el riesgo tromboembólico se utilizó la escala propuesta por Lip $\mathrm{G}$ et al ${ }^{9}$ misma que presenta un excelente valor predictivo para tromboembolismo 
en FA y que ha sido ampliamente validada por varios estudios y autores ${ }^{10-12}$. Esta nueva escala utiliza las variables insuficiencia cardiaca, hipertensión arterial, edad, diabetes, ACV previo, sexo femenino y enfermedad vascular (CHA2DS2-VASc por sus siglas en inglés). El riesgo de tromboembolia se clasifica de acuerdo al puntaje obtenido, se considera riesgo bajo a un puntaje de cero, riesgo moderado a 1 punto y un riesgo alto a puntaje de $\geq 22^{13}$.

El universo estuvo conformado por 297 historias clínicas de pacientes con diagnóstico de FA, se excluyeron 9 historias clínicas incompletas y un paciente menor de 18 años. Los resultados se analizaron mediante estadística descriptiva; para el registro y tabulación de los datos se usó: PSPP Free Software Foundation y Epidat 4.1.

\section{RESULTADOS}

Se determinó que más de la mitad de los pacientes con FA pertenecen al sexo masculino y el $70.8 \%$ de la población de estudio sobrepasa los 65 años de edad, la media fue de $70 \pm 13$ años, los datos se describen en la Tabla $N^{\circ} 1$.

\section{Tabla $N^{\circ} 1$}

Distribución de la población de estudio según edad y sexo

\begin{tabular}{crc}
\hline & $\mathbf{N ~ ( 2 9 7 )}$ & \% \\
\hline Edad (años) & & \\
$18-24$ & 1 & 0.3 \\
$25-34$ & 2 & 0.7 \\
$35-44$ & 9 & 3.0 \\
$45-54$ & 21 & 7.1 \\
$55-64$ & 54 & 18.2 \\
$65-74$ & 86 & 29.0 \\
75 o más & 124 & 41.8 \\
Sexo & & \\
Hombre & 156 & 52.5 \\
Mujer & 141 & 47.5 \\
\hline
\end{tabular}

Se evidenció que la mayoría de pacientes con FA presentan una comorbilidad, el Gráfico $\mathrm{N}^{\circ} 1$ nos muestra la frecuencia de cada una de ellas, siendo la hipertensión y la insuficiencia cardíaca las más comunes.

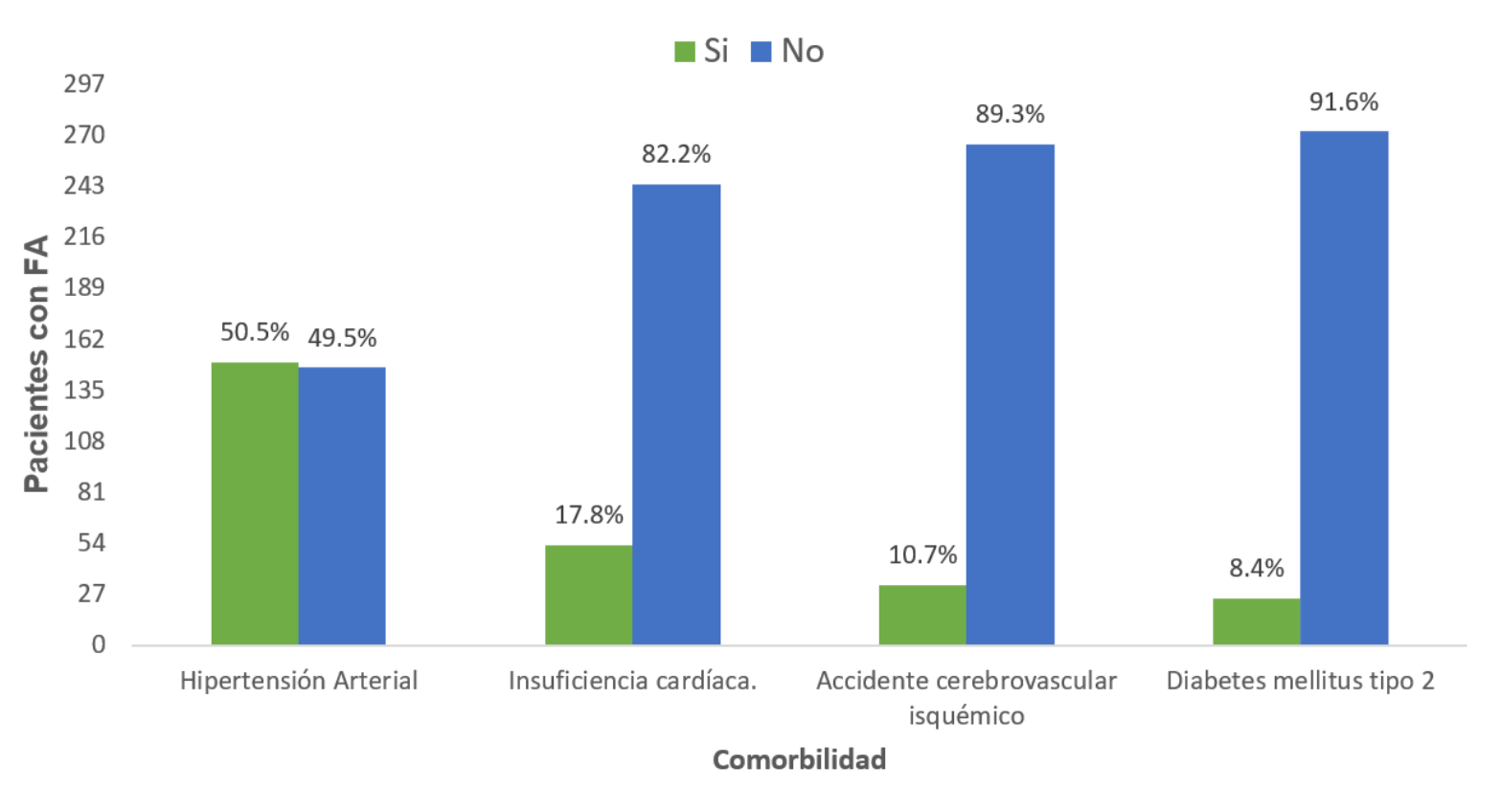

\section{Gráfico $N^{\circ} 1$}

Distribución de población de estudio según comorbilidad 
En cuanto al tipo de FA, el $82.5 \%$ de los casos fueron FA no valvular, mismos que con la escala CHAD2SD2Vasc se determinó que el $77.1 \%$ de ellos tienen un alto riesgo tromboembólico (ver Tabla $\mathrm{N}^{\circ} 2$ ).

\section{Tabla $\mathrm{N}^{\circ} 2$}

Distribución de la población de estudio según tipo de FA

\begin{tabular}{lcc}
\hline Tipo de FA & $\mathbf{n}$ & $\mathbf{\%}$ \\
Fibrilación auricular no valvular (FANV) & 245 & 82.5 \\
Fibrilación auricular valvular (FAV) & 52 & 17.5 \\
Total & $\mathbf{2 9 7}$ & $\mathbf{1 0 0}$ \\
\hline Riesgo tromboembólico en FANV & $\mathbf{n}$ & $\%$ \\
Bajo & 19 & 7.8 \\
Moderado & 37 & 15.1 \\
Alto & 189 & 77.1 \\
Total & $\mathbf{2 4 5}$ & $\mathbf{1 0 0}$ \\
\hline
\end{tabular}

En la Tabla $\mathrm{N}^{\circ} 3$ se detalla el tratamiento utilizado en pacientes con $\mathrm{FA}$, se concluye que el más empleado fue el antitrombótico, en total fueron 258 pacientes que recibían medicación y el $67 \%$ de ellos utilizó tratamiento anticoagulante, usando principalmente warfarina para este fin; en tanto el $32.6 \%$ de pacientes registraron control de ritmo y frecuencia utilizando en su mayoría betabloqueadores.

\section{Tabla $\mathrm{N}^{\circ} 3$}

Distribución de 258 pacientes en tratamiento según tipo de tratamiento que reciben

\begin{tabular}{|c|c|c|c|c|}
\hline \multicolumn{3}{|c|}{ Tratamiento Sintomático } & $\mathbf{n}$ & $\%$ \\
\hline \multirow{2}{*}{$\begin{array}{l}\text { Control aislado de la } \\
\text { frecuencia cardíaca }\end{array}$} & \multirow{2}{*}{\multicolumn{2}{|c|}{$\begin{array}{l}\text { Beta-bloqueadores* } \\
\text { Antagonistas del canal de calcio** }\end{array}$}} & 63 & 24.4 \\
\hline & & & 4 & 1.5 \\
\hline \multicolumn{3}{|l|}{ Total } & 67 & 25.9 \\
\hline \multirow{3}{*}{$\begin{array}{l}\text { Control aislado del } \\
\text { ritmo }\end{array}$} & \multirow{3}{*}{\multicolumn{2}{|c|}{$\begin{array}{l}\text { Bloqueadores de canal de potasio } \\
\text { Amiodarona } \\
\text { Cardioversión eléctrica } \\
\text { Ablación por catéter }\end{array}$}} & 1 & 0.3 \\
\hline & & & 1 & 0.3 \\
\hline & & & 1 & 0.3 \\
\hline \multicolumn{3}{|l|}{ Total } & 3 & 0.9 \\
\hline \multirow{3}{*}{ Control combinado } & \multicolumn{2}{|c|}{ Beta-bloqueadores + amiodarona } & 11 & 4.3 \\
\hline & \multicolumn{2}{|c|}{ Beta-bloqueadores + ablación } & 3 & 1.2 \\
\hline & Antagonistas & de Calcio + amiodarona & 1 & 0.3 \\
\hline \multicolumn{3}{|l|}{ Total } & 15 & 5.8 \\
\hline \multicolumn{3}{|c|}{ Tratamiento Antitrombótico } & $\mathbf{n}$ & $\%$ \\
\hline \multirow{3}{*}{$\begin{array}{l}\text { Anticoagulación ais- } \\
\text { lada }\end{array}$} & $\mathrm{AVK}^{* * *}$ & Warfarina & 29 & 11.2 \\
\hline & NACO **** & Dabigatrán & 9 & 3.5 \\
\hline & & Rivaroxabán & 3 & 1.2 \\
\hline \multicolumn{3}{|l|}{ Total } & 41 & 15.9 \\
\hline
\end{tabular}




\begin{tabular}{lllrr}
\hline $\begin{array}{l}\text { Anticoagulación } \\
\text { Combinada }\end{array}$ & Ritmo & Warfarina & 2 & 0.8 \\
& & NACO & 2 & 0.8 \\
& Frecuencia & Warfarina & 75 & 29 \\
& & NACO & 45 & 17.4 \\
& Combinado & Warfarina & 2 & 0.8 \\
& & NACO & 6 & 2.3 \\
Total & & $\mathbf{1 3 2}$ & $\mathbf{5 1 . 1}$ \\
\hline TOTAL & & $\mathbf{2 5 8}$ & $\mathbf{1 0 0}$ \\
\hline
\end{tabular}

*Atenolol, carvedilol, propanolol, metoprolol. ${ }^{* *}$ Amlodipino, diltiazem. ${ }^{* * *}$ Antagonistas de la vitamina K. ${ }^{* * * *}$ Nuevos anticoagulantes orales

\section{DISCUSIÓN}

El presente estudio sobre FA determinó que la mayoría son pacientes hombres, de la tercera edad e hipertensos. El riesgo embólico en esa población fue alto por tanto el fármaco más utilizado fue warfarina.

Los hallazgos indican que la edad media de 70 años resulta ser inferior a la de poblaciones similares, es así que, en Argentina, Fernández et al ${ }^{14}$ describen una media de 77 años de edad; la media en Perú fue de 72 años según Dávila et al $^{15}$, en tanto, Mora et $\mathrm{al}^{16}$ en España estimaron una media de 77.4 años.

El predomino del sexo masculino del $52.5 \%$ coincide con los resultados encontrados en la región, en Chile, Corbalán et $\mathrm{al}^{17}$ reportan que el $50.9 \%$ son hombres; Romero et $\mathrm{al}^{18}$ en Colombia registraron que el $57.2 \%$ de pacientes fueron del sexo masculino. Las comorbilidades identificadas en este estudio guardan relación con las reconocidas por otros autores, mismos que ratifican que la hipertensión arterial es la más frecuente. Castro et $\mathrm{al}^{6}$ en Colombia indican que el $93 \%$ de pacientes presentaron alguna comorbilidad, la hipertensión fue la más frecuente con $74.3 \%$ de casos, seguida de insuficiencia cardíaca con $53.1 \%$, de manera similar, Barón et al ${ }^{19}$ indica que el $70 \%$ de pacientes con FA tienen hipertensión arterial seguido del $26.2 \%$ con insuficiencia cardíaca.

La FA según la American Heart Association, se clasifica en valvular (FAV) y no valvular (FANV), entendiéndose como valvular cuando se encuentra asociada a estenosis mitral reumática, una válvula cardíaca mecánica o bioprotésica o una válvula mitral reparada ${ }^{20}$; esta diferenciación resulta determinante para la estratificación del riesgo tromboembólico y el manejo farmacológico de los pacientes. La FANV predomina según la tendencia reportada por varios estudios; Mantilla et al ${ }^{21}$ percibieron que $82.41 \%$ de sus pacientes tuvieron FANV, congruentemente, en El Salvador el $80.4 \%$ de los pacientes padecieron $\mathrm{FANV}^{22}$, resultados similares se obtuvieron en este estudio, en donde el $82.5 \%$ presentaron FANV y tan solo el $17.5 \%$ presentó FAV.

Mediante la aplicación de la escala CHA2DS2VASc se identificó que la mayoría de los pacientes con FANV tienen un riesgo alto de tromboembolia, el $77.1 \%$ específicamente, pero es un porcentaje menor al reportado por otros autores como Barón et al ${ }^{19}$ que indican que el $86.8 \%$ presentó un alto riesgo tromboembólico, de igual manera, Mantilla et $\mathrm{al}^{21}$ revela que el $80.4 \%$ de pacientes con FANV demostraron un riesgo elevado. Hallazgo que se justifica por la menor edad de los pacientes lo que disminuye el riesgo embólico en los mismos.

Al analizar el tratamiento que se utilizó en el Hospital José Carrasco Arteaga se evidenció que la estrategia terapéutica más usada fue la anticoagulante con el $67 \%$ usando principalmente warfarina, esto concuerda con Corbalán et $\mathrm{al}^{19}$ que en Chile describen que el $81.9 \%$ de pacientes con FA son tratados con terapia anticoagulante y de ellos el $70 \%$ reciben antagonistas de la vitamina $\mathrm{K}$; en contraste, la tendencia en Colombia indica que de los pacientes con anticoaguloterapia, el $41.2 \%$ utiliza los nuevos anticoagulantes orales (NACO) y solo el $23.9 \%$ utiliza warfarina ${ }^{6}$, es de resaltar que estudios recientes respaldan esta última tendencia puesto que en los pacientes tratados con NACO como dabigatrán aumentan los años de vida comparado con la warfarina ${ }^{23,24}$. 
Las terapias para el control del ritmo y la frecuencia en la localidad resultan ser similar a la observada en otros países, es así que el $25.9 \%$ de los pacientes usaron betabloqueadores (atenolol y carvedilol) para el control de frecuencia y el $5.9 \%$ de pacientes usó tratamiento combinado para ritmo y frecuencia (betabloqueantes más amiodarona) lo que coincide con México puesto que, para el control de la frecuencia el $56 \%$ de los casos usan betabloqueantes y los antiarrítmicos clase III fueron para el control del ritmo $^{25}$, de igual manera en Uruguay el $37 \%$ de casos usa betabloqueantes ${ }^{26}$.

\section{CONCLUSIONES}

Al caracterizar a los pacientes con FA del Hospital José Carrasco Arteaga de la ciudad de Cuenca durante el año 2017, se encuentran semejanzas en relación a otras poblaciones, con predominio del sexo masculino y un alto porcentaje son de la tercera edad. La FA está fuertemente asociada a hipertensión arterial y a un alto riesgo embólico por las múltiples patologías coexistentes. La estrategia terapéutica más usada fue la anticoagulante, siendo la warfarina el fármaco más utilizado para este fin. Los datos aportados en este estudio pueden servir de base para otros enfocados en la prevención y tratamiento de esta patología con alta prevalencia en Cuenca.

\section{ASPECTOS BIOETICOS:}

La información obtenida en este estudio fue administrada con absoluta reserva, su uso fue únicamente en función de la investigación. Se obtuvo autorización por escrito del coordinador general de investigación del Hospital José Carrasco Arteaga.

\section{INFORMACIÓN DE LOS AUTORES}

- Balarezo Arias Fernando Xavier. Médico. Libre ejercicio. Cuenca - Azuay - Ecuador. e-mail: balarezo.fernando@hotmail.com ORCID: https://orcid.org/0000-0002-7469-0791

- Anilema Cóndor Raúl Daniel. Médico. Libre ejercicio. Cuenca - Azuay - Ecuador. e-mail: daniel_rdac@hotmail.com ORCID: https://orcid.org/0000-0003-2516-0118

\section{CONTRIBUCIÓN DE AUTORES}

Los autores declaran haber contribuido de forma similar en el levantamiento, procesamiento de la información, análisis estadístico, diseño y elaboración del presente artículo.

\section{CONFLICTO DE INTERESES}

Los autores declaran no tener conflictos de intereses.

\section{FUENTES DE FINANCIAMIENTO}

Fondos propios.

\section{AGRADECIMIENTO}

Un agradecimiento especial a la Dra. Zoila Elizabeth Verdugo Carrión por su valiosa contribución en el desarrollo del presente artículo.

\section{BIBLIOGRAFÍA}

1. Organización Mundial de la Salud. [online]; 2017 [citado 15 octubre 2020] Disponible en: https://www.who.int/es/news-room/factsheets/detail/cardiovascular-diseases-(cvds)

2. Ramírez-Barrera JD, Agudelo-Uribe JF, Correa-Velásquez R, González-Rivera E. Fisiopatología de la fibrilación auricular. Rev Colomb Cardiol. 2016;23(5):9-14. DOI: 10.1016/j.rccar.2016.10.004. Disponible en: http://linkinghub.elsevier.com/retrieve/pii/ S0120563316301590

3. Mahmood S, Levy D, Vasan R S, Wang, T. The Framingham Heart Study and the epidemiology of cardiovascular disease: a historical perspective. The Lancet. 2014; 383(9921):999-1008. DOI: 10.1016/s01406736(13)61752-3. Disponible en: https:// www.thelancet.com/journals/lancet/article/ PIIS0140-6736(13)61752-3/fulltext

4. Gómez-Doblas JJ, López-Garrido MA, Esteve-Ruiz I, Barón-Esquivias G. Epidemiología de la fibrilación auricular. Rev Esp Cardiol Supl. 2016;16(1):2-7. DOI: $\quad$ 10.1016/S1131-3587(16)30007-3. Disponible en: https://www.revespcardiol. org/es-epidemiologia-fibrilacion-auriculararticulo-S1131358716300073 
5. Lakshminarayan K, Anderson DC, Herzog CA, Qureshi Al. Clinical Epidemiology of Atrial Fibrillation and Related Cerebrovascular Events in the United States. Neurologist. 2018;4(3):143-150. DOI: 10.1097/ NRL.0b013e31815cffae Disponible en: https://www.ncbi.nlm.nih.gov/pmc/articles/ PMC5619693/pdf/nihms114055.pdf

6. Castro-Clavijo J, Quintero S, Valderrama F, DiaztagleJ, Ortega J.Prevalencia defibrilación auricular en pacientes hospitalizados por Medicina Interna. Rev Colom Cardiol. 2020;27(6):560-566. DOI:10.1016/j. rccar.2019.01.007.Disponible en: https:// rccardiologia.com/previos/RCC\%20 2020\%20Vol.\%2027/RCC_2020_27_6_ NOV-DIC/RCC_2020_27_6_560-566.pdf

7. Carlos M, González-Galeano C. Fibrilación auricular en un hospital del interior de Paraguay. Rev Nac (Itaugua). 2015;7(2):2023. DOI: biblio-884775 . Disponible en: https://pesquisa.bvsalud.org/portal/resource/ pt/biblio-884775?lang $=f r$

8. Pérez-Copete J, Esteve-Pastor M, Roldán V, Valdés M, Marín F. Escalas de evaluación del riesgo tromboembólico y hemorrágico en la fibrilación auricular. Rev Española Cardiol Supl. 2016;16(Supl.A):25-32. DOI: 10.1016/S1131-3587(16)30011-5 Disponible en: http://linkinghub.elsevier.com/retrieve/pii/ S1131358716300115

9. Lip G, Nieuwlaat R, Pisters R, Lane D, Crijns H. Refining Clinical Risk Stratification for Predicting Stroke and Thromboembolism in Atrial Fibrillation Using a Novel Risk FactorBased Approach. Chest. 2010;137(2):26372. DOI: 10.1378/chest.09-1584 Disponible en: https://linkinghub.elsevier.com/retrieve/ pii/S0012369210600670

10. Olesen J, Lip G, Hansen P, Tolstrup J, Selmer C, Ahelhoff O, Olsen A, Gislason G, Pedersen C. Validation of risk stratification schemes for predicting stroke and thromboembolism in patients with atrial fibrillation: nationwide cohort study. BMJ. 2011; 342:d124. DOI: https://doi.org/10.1136/bmj.d124

11. Coppens M, Eikelboom JW, Hart RG, Yusuf S, Lip GY, Dorian P, Shestakovska O, Connolly SJ. The CHA2DS2-VASc score identifies those patients with atrial fibrillation and a CHADS2 score of 1 who are unlikely to benefit from oral anticoagulant therapy. Eur Heart J. 2013 Jan;34(3):170-6. DOI: 10.1093/ eurheartj/ehs314. Epub 2012 Sep 27.

12. Kornej J, Hindricks G, Kosiuk J, Arya A, Sommer P, Husser D, Rolf S, Richter S, Huo Y, Piorkowski C, Bollmann A. Comparison of CHADS2, R2CHADS2, and CHA2DS2-VASc scores for the prediction of rhythm outcomes after catheter ablation of atrial fibrillation: the Leipzig Heart CenterAF Ablation Registry. Circ Arrhythm Electrophysiol. 2014 Apr;7(2):2817. DOI: 10.1161/CIRCEP.113.001182.

13. Voukalis C, Lip GYH, Shantsila E. Emerging Tools for Stroke Prevention in Atrial Fibrillation. EBIOM. 2016;15(4):26-39.DOI: 10.1016/j.ebiom.2016.01.017. Disponible en: http://www.ebiomedicine.com/article/S23523964(16)30013-5/

14. Fernández $\mathrm{G}$, Maid $\mathrm{G}$, Arias $\mathrm{A}$, Maldonado S, Pizarro R, Belziti C. Prevalencia de fibrilación auricular y factores predictores de su aparición en pacientes portadores de marcapos bicamerales. Rev Colom Cardiol. 2016;86(3):214-220.DOI: 10.1016/j. acmx.2016.04.008 Disponible en: http://www. scielo.org.mx/pdf/acm/v86n3/1405-9940acm-86-03-00214.pdf

15. Dávila-Hernández C, Carranza-Quispe F. Características clínicas y epidemiológicas de la fibrilación auricular en pacientes del Hospital Regional de Ica. Rev Soc Peru Med Interna. 2018;31(2):45-49.DOI: 10.36393/ spmi.v31i2.31. Disponible en: http:// revistamedicinainterna.net/index.php/spmi/ article/view/31

16. Mora-Llabata $V$, Dubois-Marqués $D$, RoldánTorres I, Mateu-Navarro C, Sanz-García J, Moreno-Ballester V, Mira-Gimeno S, Albiñaña-Fernandez $F$. Prevalencia de fibrilación auricular y características de la fibrilación auricular no valvular en la población general. Registro AFINVA. Rev Colom Cardiol. 2017;24(1):26-23.DOI: 10.1016/j. rccar.2016.03.021 Disponible en: http://www. scielo.org.co/pdf/rcca/v24n1/0120-5633rcca-24-01-00026.pdf

17. Corbalán R, Conejeros C, Rey C, Stockins B, Eggers G, Astudillo C. Características basales, manejo de terapias antitrombóticas y pronóstico de pacientes chilenos con FA no valvular. Lecciones del Registro 
GARFIELD AF en Chile. Rev. méd. Chile. 2017;145(8):963-971.DOI: 10.4067/s003498872017000800963. Disponible en: https:// scielo.conicyt.cl/scielo.php?script=sci_ arttext\&pid=S0034-98872017000800963\&In $\mathrm{g}=\mathrm{en} \& \mathrm{nrm}=$ iso\&tlng=en

18. Romero M, Chávez D. Carga de enfermedad atribuible a fibrilación auricular en Colombia (2000-2009). Rev. Colomb. Cardiol. 2014. 21(6):374-381. DOI: 10.1016/j. rccar.2014.08.006. Disponible en: https:// rccardiologia.com/previos/R C C $\% 20$ 2014\%20Vol.\%2021/RCC_2014_21_6_ NOV-DIC/RCC_2014_21_6_374-381.pdf

19. Barón-Esquivias G, Gómez F, Amo C, Sainz I, Guisado A, Adriaensens A. Características clínicas y manejo de la fibrilación auricular en las consultas de cardiología y su adecuación a las guías europeas de 2010. Cardiocore. 2012;47(1):30-36.DOI:10.1016/j. carcor.2011.07.002 Disponible en: https:// www.sciencedirect.com/science/article/abs/ pii/S1889898X11001095?via\%3Dihub

20. Martins R, Galand V, Colette E, Behar N, Pavin D, Leclercq C, Daubert J, Mabo P. Defining nonvalvular atrial fibrillation: A quest for clarification. Am Heart. 2016;178:161-167. DOI:10.1016/j.ahj.2016.05.014 Disponible en: http://www.ncbi.nlm.nih.gov/pubmed/27502864

21. Mantilla-Villabona L, Ospina-Galeano D, Gutiérrez-Ortiz A, Camacho P. Pacientes con fibrilación auricular atendidos en consulta de atención primaria de una institución de alta complejidad. Rev Colom Cardiol. 2018; 25(2):124-130.DOI: 10.1016/j. rccar.2017.09.005 Disponible en: https:// www.sciencedirect.com/science/article/pii/ S0120563317302097

22. Merino S, Mercedes J, Landaverde $\mathrm{H}$, et al. Caracterización de la fibrilación auricular en el Servicio de Medicina Interna del Hospital Nacional San Rafael (El Salvador). CorSalud. 2016;8(1):8-18. Disponible en: http://www. revcorsalud.sld.cu/index.php/cors/article/ view/92/238

23. Savarese G, Giugliano R, Rosano G, Mcmurray J, Magnani G, Filippatos G, Santo D, Lars B,Perrone-Filardi P. Efficacy and Safety of Novel Oral Anticoagulants in Patients With Atrial Fibrillation and Heart Failure A Meta-Analysis. J Am Coll Cardiol
HF.2016;4(11):870-880. DOI:10.1016/j. jchf.2016.07.012 Disponible en: https://www. jacc.org/doi/pdf/10.1016/j.jchf.2016.07.012

24. Camm A. The RE-LY study: Randomized Evaluation of Long-term anticoagulant therapY: dabigatran vs. warfarin. Eur Heart J. 2009. 1;30(21):2554-2555. DOI: 10.1093/eurheartj/ehp384 Disponible en: https://academic.oup.com/eurheartj/ article/30/21/2554/518562

25. Lara-Vaca S, Cordero-Cabra A, MartínezFlores E, Iturralde-Torres P. Registro Mexicano de Fibrilación Auricular (ReMeFa). Gac Med Mex. 2014,150(Sup1):48-59. Disponible en: https://www.anmm.org.mx/ GMM/2014/s1/GMM_150_2014_s1_048059.pdf

26. Zoni Berisso Massimo. Fibrilación auricular: un importante problema de salud pública. Rev Urug Cardiol. 2015;30(3):347-356. Disponible en: https://www.redalyc.org/ articulo.oa?id=479747285012 